\title{
Módulos embebidos en micro-tecnología FPGA de modelo estocástico de primer orden
}

\author{
Karen Alicia Aguilar Cruz, Romeo Urbieta Parrazales, \\ José Antonio Flores Escobar, Midory Esmeralda Vigueras Velázquez, \\ José de Jesús Medel Juárez \\ Instituto Politécnico Nacional, Centro de Investigación en Computación, \\ Ciudad de México, México \\ Karen_ali320@hotmail.com, \\ rurbieta700@gmail.com,jafloresescobar@hotmail.com, \\ midory.vigueras@gmail.com, jjmedelj@gmail.com
}

\begin{abstract}
Resumen. En este artículo se describe un filtro digital diseñado en etapas y embebido en arreglo de compuertas de campo (FPGA) basado en el segundo momento de probabilidad y en el modelo autorregresivo de medias móviles (ARMA $(1,1)$ ) de primer orden; ambos en sus formas recursivas y no recursivas. Los modelos se describen como módulos a bloques para representar las variables de estimación e identificación, así como las variables de medición del proceso a la entrada y salida del sistema tipo caja negra, el cual representa a un proceso de energía eólica. El filtro estocástico es programado en lenguaje de descripción de hardware Verilog ${ }^{\circledR}$, brindando una implementación en estimación digital. Para la verificación de su estructura se obtuvo el acoplamiento de los módulos considerados en el filtrado estocástico de primer orden.
\end{abstract}

Palabras clave: filtro digital estocástico, estimación, identificación, ARMA.

\section{Embedded Modules in Micro-technology FPGA of the First Order Stochastic Model}

\begin{abstract}
This article describes a digital filter designed in stages and embedded in field gate arrays (FPGA) based on the second probability moment and in the autoregressive model of moving averages (ARMA $(1,1)$ ) of first order; both in their recursive and non-recursive forms. The models are described as block modules to represent the estimation and identification variables, as well as the process measurement variables at the input and output of the black box system, which represents a wind energy process. The stochastic filter is programmed in Verilog $®$ hardware description language, providing an implementation in digital estimation. For the verification of its structure, the coupling of the modules considered in the first-order stochastic filtering was obtained.
\end{abstract}

Keywords: stochastic digital filter, estimation, identification, ARMA. 


\section{Introducción}

La problemática que se encuentra en el diseño de estimadores digitales es encontrar el parámetro de innovación dados los datos medidos de un sistema de caja negra (BB, por sus siglas en inglés Black Box) de primer orden para determinar los estados estimados mediante algún criterio [1]. Lo anterior requiere la descripción de tres modelos estocásticos, descritos a continuación.

Primero, el modelo que representa al proceso de estudio a través de un modelo lineal no variante en el tiempo (LTI, por sus siglas en inglés Linear Time-Invariant) de primer orden, conteniendo la descripción de los estados internos a priori del proceso mediante el modelo lineal $\tilde{x}_{k+1}=a \tilde{x}_{k}+b \widetilde{w}_{k}$, donde, $k=0,1,2, \ldots, n$ representa los $n$ muestreos, $a$ y $b$ son constantes tales que $a$ y $b \in \mathfrak{R}$, y $\widetilde{w}_{k}$ es ruido blanco con media y variancia definidas, $\widetilde{w}_{k} \in N\left(\mu \approx 0, \sigma^{2}<\infty\right)$ [2]. En esta notación, la tilde sobre las variables significa que son aleatorias. Si al modelo LTI se le hace pasar por un retardo se logra obtener el estado lineal $\tilde{x}_{k}$.

Segundo, el modelo del sensor lineal, que viene ubicado a la salida del modelo del proceso lineal para leer los estados internos $\tilde{x}_{k}$, caracterizado por una serie de mediciones o datos digitales de salida, representadas por $\tilde{y}_{k}=c \tilde{x}_{k}+d \tilde{v}_{k}$, donde $k=$ $0,1,2, \ldots . n$, y $\tilde{v}_{k}$, ruido de salida con características de media y varianza definidas.

Tercero, el modelo del estimador estocástico lineal, el cual tiene como entrada las mediciones efectuadas por el sensor $\tilde{y}_{k}$, y como función el criterio de estimación empleado para determinar el parámetro estimado, o parámetro de innovación, $\hat{a}_{k}$ y los estados estimados deseados $\hat{x}_{k}$. La notación ( $(\widehat{)})$ significa que los parámetros y variables son estimados.

De los criterios existentes en la teoría estocástica [3] para la estimación de parámetros, se considera el segundo momento de probabilidad estocástica. Este criterio se basa en los espacios de Hilbert $\left\langle\tilde{y}_{k}, \tilde{y}_{k-1}>\right.$ usando los operadores de la probabilidad estocástica: media $\left(\mathrm{E}\left\{\tilde{y}_{k}\right\}=0\right)$, varianza $\left(\operatorname{var}\left\{\tilde{y}_{k}\right\}=\sigma^{2}\right)$ y covarianza $\left(\operatorname{cov}\left\{\tilde{y}_{k}, \tilde{y}_{k+t}\right\}=0\right)$.

Por otra parte, para la elección de los sistemas digitales embebidos, ya sea en una sola pastilla o chip, para aplicaciones en filtros, control automático o procesamiento de señales, el análisis de las características eléctricas, mecánicas, y de programación juega un papel importante en la búsqueda de sistemas que sean eficientes, confiables e incluso de innovación para el diseño. Por ejemplo, dentro de las características eléctricas se encuentran la capacidad de memoria, la velocidad de procesamiento, el número de puertos de entrada y salida (E/S) y el número de bloques de programación; mientras que en las características mecánicas se encuentran las dimensiones, la forma, el material empleado, el acabado, etc. Para el caso de la programación, se verifica que cuenten con un conjunto de instrucciones acorde a la solución del problema, así como facilidad para borrar y grabar información.

En este trabajo, para la implementación del filtro, se seleccionó el sistema modular digital de Arreglos de Compuertas Programables en Campo (FPGA, por sus siglas en inglés Field-Programmable Gate Array), cuyas características eléctricas incluyen una alta velocidad de procesamiento, la cantidad de puertos de $\mathrm{E} / \mathrm{S}$ y de bloques funcionales de programación, así como memoria y robustez a perturbaciones del ambiente [4-6]. 


\section{Método}

\subsection{Modelo digital ARMA estocástico}

El modelo digital ARMA estocástico se expresa mediante dos ecuaciones lineales definidas en (1) y (2). La ecuación (1) de incremento de estados $x_{k+1}$, incluye una suma de productos que representan al parámetro $a$ que se desea conocer, multiplicado por el estado interno $\tilde{x}_{k} \mathrm{y}$, al sistema de excitación $\widetilde{w}_{k}$ multiplicado por un factor constante $b$. La variable medida por el sensor $\tilde{y}_{k}$, definida en (2) corresponde al producto del parámetro $c$ y el estado interno correspondiente $\tilde{x}_{k}$, más una perturbación aleatoria $\tilde{v}_{k}$ de salida del sistema, por una constante $d$. En este caso, el sistema de estudio es considerado como un modelo BB caracterizado por los dos ruidos $\widetilde{w}_{k}$ y $\tilde{v}_{k}$ :

$$
\begin{gathered}
\tilde{x}_{k+1}=a . * \tilde{x}_{k}+b . * \widetilde{w}_{k}, \\
\tilde{y}_{k}=c . * \tilde{x}_{k}+d . * \tilde{v}_{k} .
\end{gathered}
$$

Observando las ecuaciones (1) y (2) se ven cuatro operaciones de multiplicación y dos de sumas. Usando estas dos expresiones matemáticas y simbología de bloques de circuitos digitales, se puede construir el modelo digital ARMA mostrado en la Fig. 1, seguido de su desarrollo en modelo digital en lenguaje de descripción de hardware Verilog®, Fig. 2. En este modelo, se observan los parámetros de medición y las variables de estados, considerando que los ruidos E/S cuentan con una distribución gaussiana.

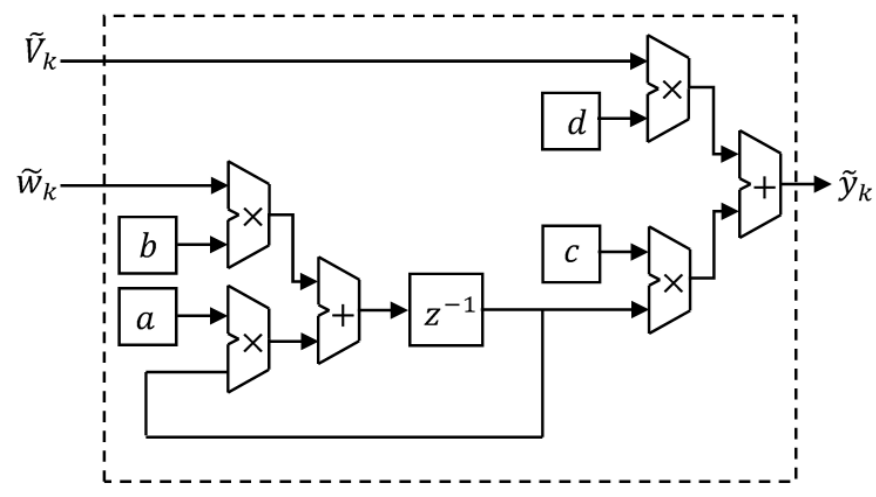

Fig. 1. Módulo en bloques del modelo digital ARMA estocástico. 


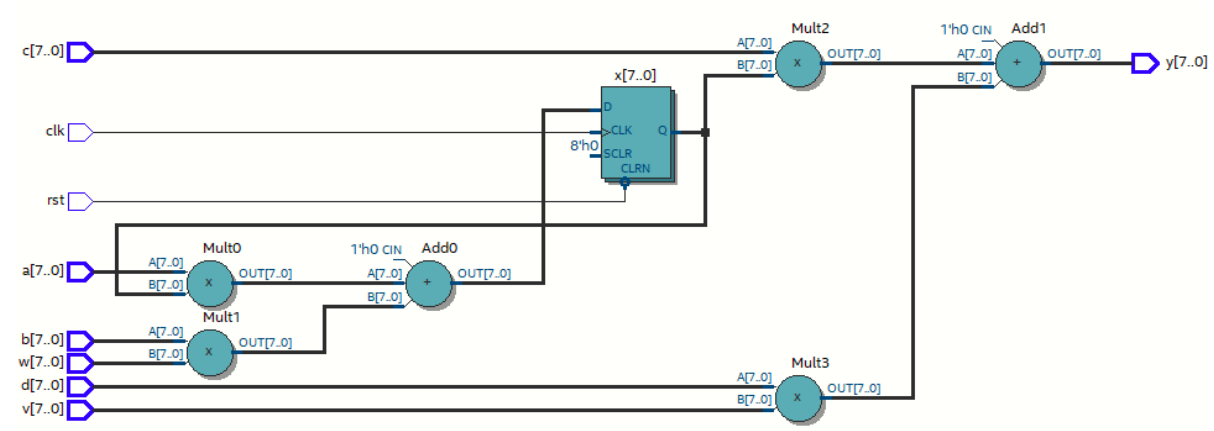

Fig. 2. Representación del modelo ARMA estocástico en un módulo digital Verilog®.

\subsection{Modelo digital recursivo estocástico}

La estimación recursiva consiste en la estimación secuencial del modelo ARMA para distintos tamaños muestrales. Dependiendo del valor de la muestra " $k$ " que se emplee, es posible determinar la convergencia o estabilidad del modelo. Si el valor de la muestra es grande entonces hay cambio radical en la estimación del parámetro y por consecuencia los estados se pueden salir de entre los valores cero y uno, produciendo de esta manera una mala convergencia e inestabilidad. La idea es entonces que si no hay cambio estructural se espera que las estimaciones de los parámetros se mantengan constantes al ir aumentando la muestra en forma secuencial. A continuación, en las ecuaciones (3) y (4) se presenta el modelo recursivo estocástico a partir del modelo ARMA, incluyendo la definición de ruidos, y su representación digital:

$$
\begin{gathered}
\tilde{y}_{k}=a . * \tilde{y}_{k-1}+\tilde{\bar{V}}_{k}, \\
\tilde{V}_{k}=-a . * d . * \tilde{v}_{k-1}+c . * b . * \widetilde{w}_{k-1}+d * \tilde{v}_{k},
\end{gathered}
$$

donde $\tilde{\bar{y}}_{k}$ es salida recursiva y $\tilde{\bar{V}}_{k}$ es la mezcla de ruidos blancos. En la Fig. 3 se muestra el módulo digital de estas variables y su módulo digital de descripción de hardware en la Fig. 4.

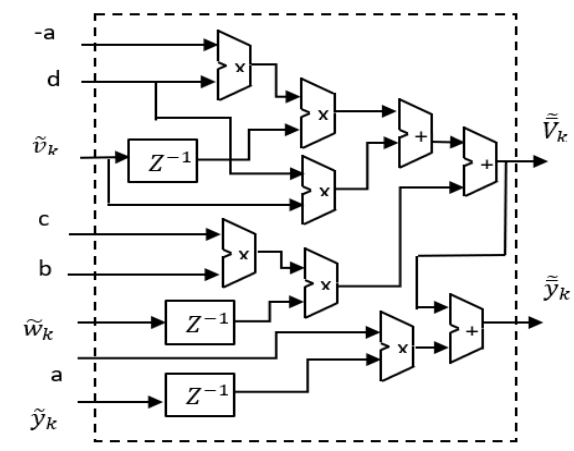

Fig. 3. Módulo en bloques del modelo digital recursivo estocástico. 


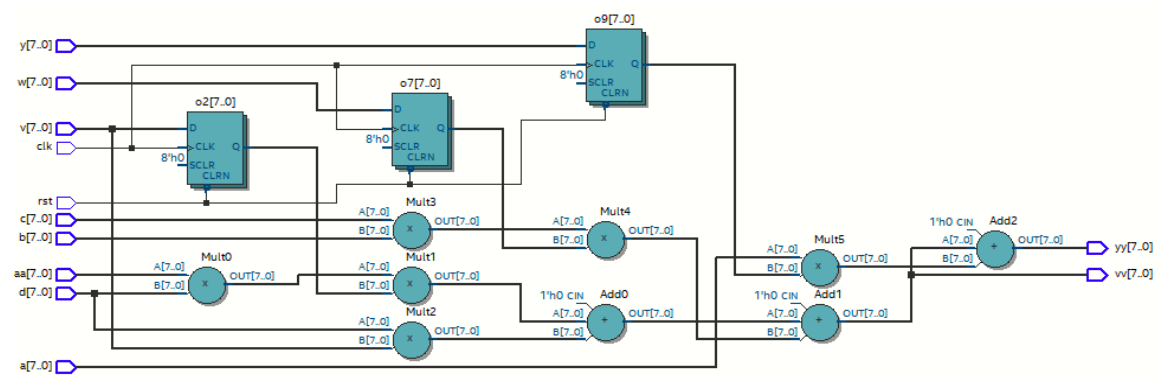

Fig. 4. Módulo digital Verilog® del modelo digital recursivo estocástico $\tilde{\bar{y}}_{k}$.

\subsection{Modelo digital recursivo de la varianza $\widetilde{p}_{k}$}

La varianza [7] $\tilde{p}_{k}$ del modelo estocástico, descrita en la ecuación (5), se presenta como una función de los muestreos digitales $\mathrm{k}$, y dos sumandos. El primer sumando es una multiplicación de salidas $\tilde{\bar{y}}_{k} \tilde{y}_{k-1}$ y el segundo una convolución entre los $\mathrm{k}$ muestreos y la propia función $\tilde{p}_{k-1}$ retardadas por un periodo de muestreo:

$$
\tilde{p}_{k}=\frac{1}{k} \sum_{1}^{n} \tilde{\bar{y}}_{k} \tilde{y}_{k-1}=\frac{1}{k}\left(\tilde{\bar{y}}_{k} \tilde{y}_{k-1}+(k-1) \tilde{p}_{k-1}\right) .
$$

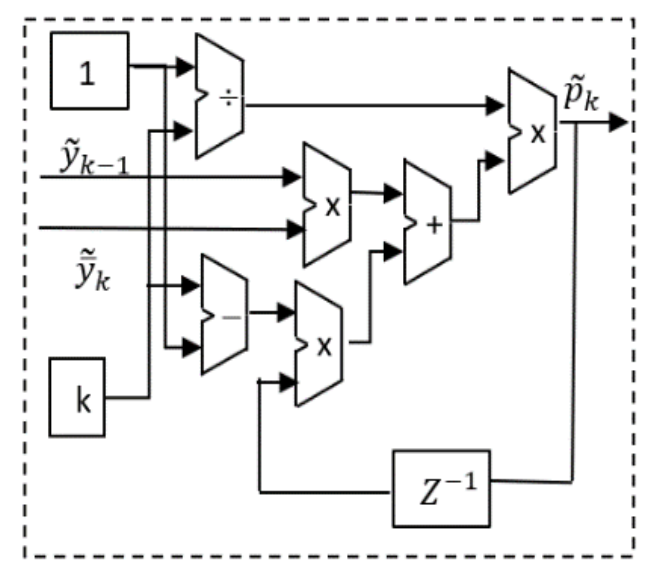

Fig. 5. Material del Modelo digital de la Varianza estocástica $\tilde{p}_{k}$.

En las Figs. 5 y 6 se muestran tanto el sistema digital en bloques que puede determinar la varianza $\tilde{p}_{k}$, así como el diagrama de implementación en Verilog®, tal como se efectuó en el primer módulo. 


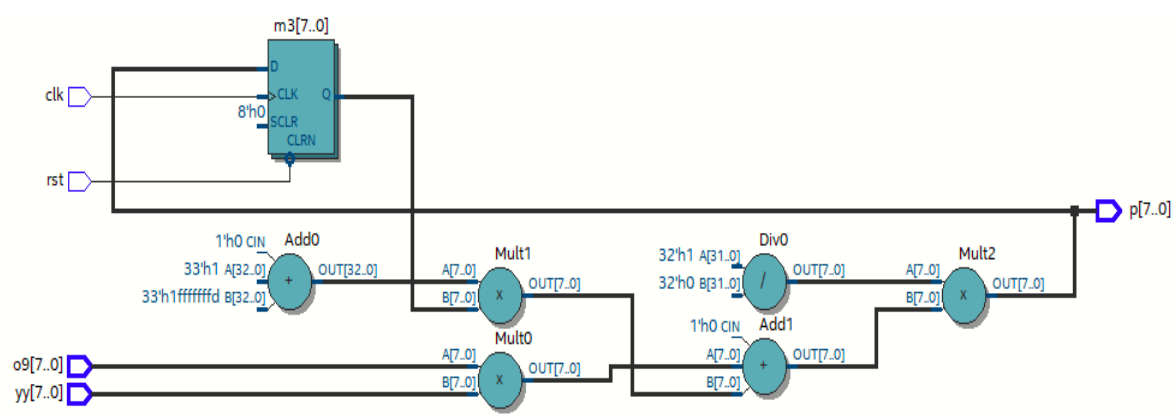

Fig. 6. Material del Módulo digital Verilog $5^{\circledR}$ del modelo digital estocástico $\tilde{p}_{k}$.

\subsection{Modelo digital de la covarianza $\widetilde{\boldsymbol{q}}_{k}$}

La ecuación (6) muestra el término que permite obtener la covarianza [7] $\tilde{q}_{k}$ expresada en dos sumatorias que representan dos secuencias. La primera con la salida misma y la segunda con la respuesta y ruido de salida con un retardo.

$$
\tilde{q}_{k}=\frac{1}{k}\left(\sum_{1}^{n} \tilde{y}_{i-1}^{2}-d \sum_{1}^{n} \tilde{v}_{i-1} \tilde{y}_{i-1}\right) \text {. }
$$

Desarrollando las sumatorias se llega a (7):

$$
\tilde{q}_{k}=\frac{1}{k}\left(\tilde{y}_{k-1}^{2}-d * \tilde{v}_{k-1} \tilde{y}_{k-1}+(k-1) \tilde{q}_{k-1}\right) \text {. }
$$

La representación de la obtención de la covarianza se muestra en diagrama a bloques en la Fig. 7 y de forma implementada en Verilog®, en la Fig. 8.

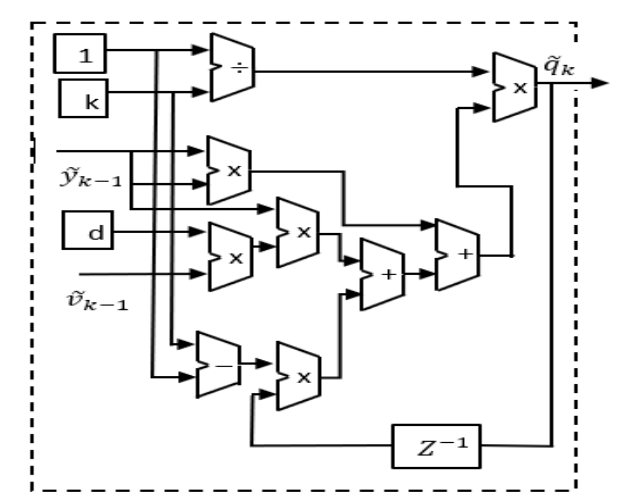

Fig. 7. Modelo digital en bloques de la covariancia $\widetilde{\boldsymbol{q}}_{\boldsymbol{k}}$. 


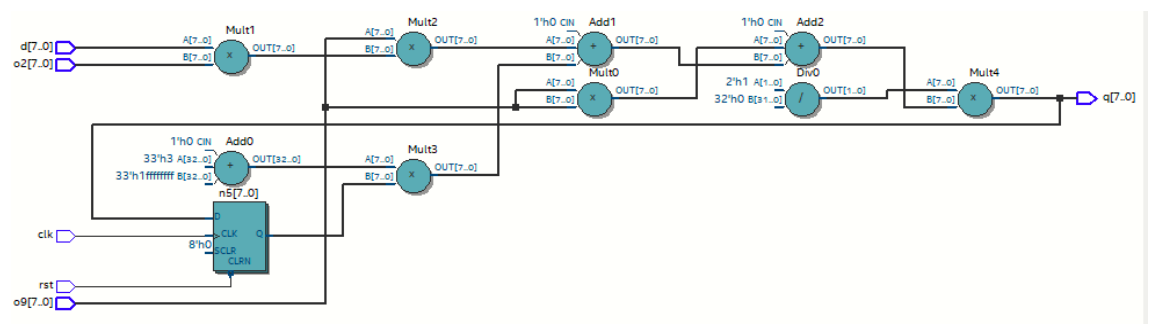

Fig. 8. Módulo en Verilog® del modelo de la Covarianza $\widetilde{\boldsymbol{q}}_{\boldsymbol{k}}$.

\section{Resultados}

\subsection{Modelo digital de estimación $\widetilde{\boldsymbol{a}}_{\boldsymbol{k}}$}

El modelo de estimación estocástica viene representado por el segundo momento de probabilidad estocástica, donde la ecuación (3) se convoluciona con $\tilde{y}_{k-1}$, i.e. $\xi\left\{\tilde{\bar{y}}_{k} \tilde{y}_{k-1}\right\}$, obteniendo el parámetro estimado $\tilde{a}_{k}$ como en la ecuación (8).

$$
\tilde{a}_{k}=\frac{\xi\left\{\tilde{y}_{k} \tilde{y}_{k-1}\right\}}{\xi\left\{\tilde{y}_{k-1}^{2}-d * \tilde{v}_{k-1} \tilde{y}_{k-1}\right\}} .
$$

Desarrollando (8) se obtiene el parámetro estimado (9), descrito como el cociente entre la varianza y la covarianza.

$$
\tilde{a}_{k}=\frac{\tilde{p}_{k}}{\tilde{q}_{k}}=\frac{\left(\tilde{\bar{y}}_{k} \tilde{y}_{k-1}+(k-1) \tilde{p}_{k-1}\right)}{\tilde{y}_{k-1}^{2}-d * \tilde{v}_{k-1} \tilde{y}_{k-1}+(k-1) \tilde{q}_{k-1}}
$$

donde $\tilde{a}_{k} \in \mathfrak{R} \cdot\{0,1\}$ es el parámetro digital estimado en función de $\mathrm{k}$ muestreos $\{1,2$, $\ldots, \mathrm{n}\}, \mathrm{y} \tilde{p}_{k}, \tilde{q}_{k} \in \mathfrak{R}\{0,1\}$ son la varianza y covarianza del modelo estocástico bajo el método del segundo momento.

Finalmente, en la Fig. 9 se tiene la integración de los módulos descritos anteriormente y en la Fig. 10, se aprecia el sistema de filtro digital estocástico que se grabará en el sistema digital de desarrollo basado en tecnología FPGA.

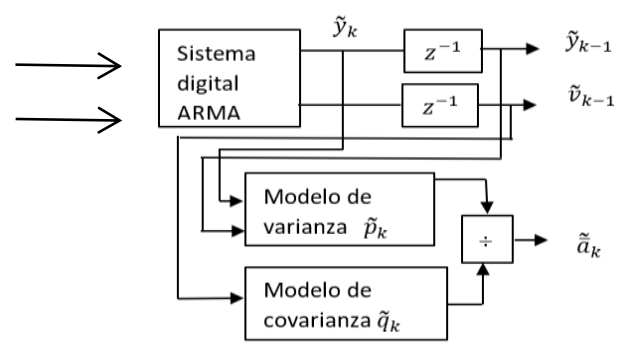

Fig. 9. Modelo digital de filtro digital de estimación e identificación basado en el segundo momento de probabilidad estocástica. 


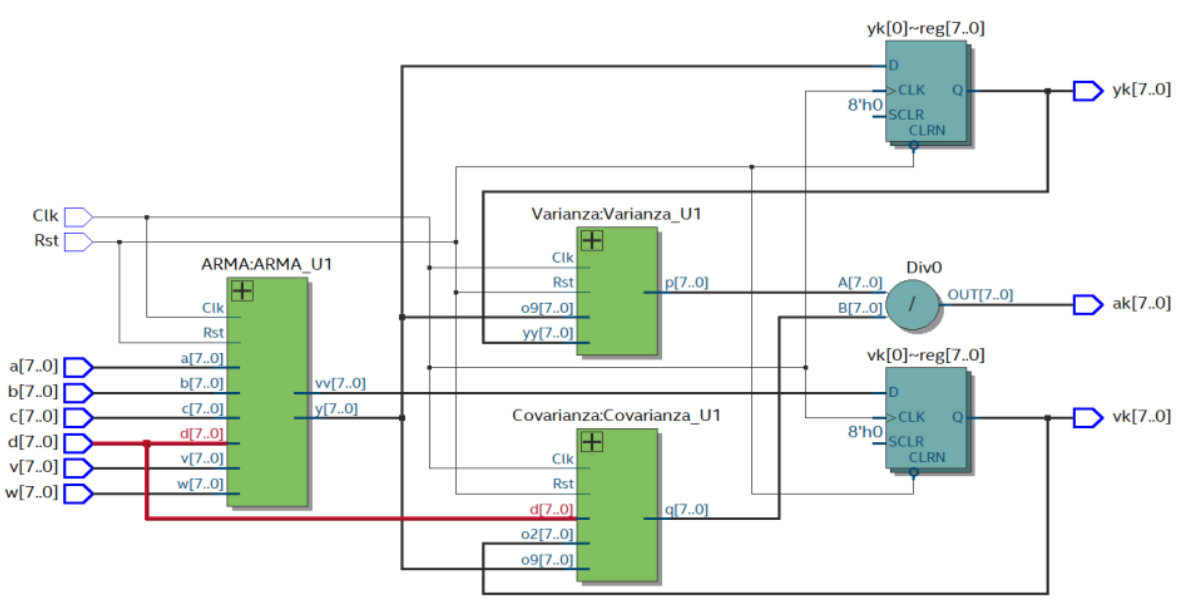

Fig. 10. Material del módulo Verilog® del modelo digital de estimación e identificación basado en el segundo momento de probabilidad estocástica.

\section{Conclusiones}

El presente trabajo presentó el método de estimación de parámetros e identificación de las variables de salida de un sistema, basado en el segundo momento de probabilidad estocástica, utilizando una notación discreta para el modelo digital estocástico que emula al modelo de primer orden de caja negra y a los parámetros de datos obtenidos en las mediciones de su entrada y salida.

El modelo estocástico para la caja negra se determinó a través de un modelo ARMA $\mathrm{y}$, mezclando las ecuaciones de las variables que contienen los ruidos de $w_{k}, v_{k}$ de E/S se pudo llegar a un modelo ARMA recursivo de la variable de identificación. Esto con la idea de implementarlo en un sistema digital como es sistema de desarrollo FPGA de Altera. Mientras que para la descripción de la estimación se hizo uso de los conceptos de esperanza matemática, varianza y covarianza.

Como resultado, el parámetro de innovación $\tilde{\bar{a}}_{k}$ estocástica resultó en un cociente entre la varianza y covarianza $P_{k}, Q_{k}$, respectivamente. El resultado fue la implementación del sistema de filtrado estocástico e identificación en un sistema digital usando FPGA de Altera, brindando una herramienta que contiene los bloques de estimación basados en operadores estocásticos matemáticos y que es de gran utilidad en aplicaciones prácticas de filtrado lineal estocástico.

Agradecimientos. Los autores agradecen al Centro de Investigación en Computación del Instituto Politécnico Nacional (IPN) y al Consejo Nacional de Ciencia y Tecnología (CONACyT), por su apoyo en el desarrollo de este trabajo, a través de los proyectos SIP20180762 y SIP20181910. 


\section{Referencias}

1. Mendel, J. M.: Lessons in estimation theory for signal processing, communications, and control. Pearson Education (1995)

2. Medel Juárez, J. J., García Infante, J. C., Urbieta Parrazales, R.: Identificador con comparación entre dos estimadores. Revista Mexicana de Física, 57(5), pp. 414-420 (2011)

3. Kumar, P. R., Varaiya, P.: Stochastic systems: Estimation, identification, and adaptive control (Vol. 75). SIAM (2015)

4. Xia, D. Z., Hu, Y. W., Kong, L.: Adaptive Kalman filtering based on higher-order statistical analysis for digitalized silicon microgyroscope. Measurement, 75, pp. 244-254 (2015)

5. Sass, R., Schmidt, A. G.: Embedded systems design with platform FPGAs: principles and practices. Morgan Kaufmann (2010)

6. Gazzano, J. D. D., Crespo, M. L., Cicuttin, A., Calle, F. R.: Field-Programmable Gate Array (FPGA) Technologies for High Performance Instrumentation. IGI Global (2016)

7. Evans, M. J., Rosenthal, J. S.: Probabilidad y estadística. Reverté (2005) 